\title{
Bridging gaps between several forms of granular computing
}

\author{
Didier Dubois $^{1} \cdot$ Henri Prade ${ }^{1}$
}

Received: 23 July 2015/ Accepted: 15 October 2015/Published online: 5 January 2016

(C) Springer International Publishing Switzerland 2015

\begin{abstract}
Two important ideas at the core of Zadeh's seminal contributions to fuzzy logic and approximate reasoning are the notions of granulation and that of possibilistic uncertainty. In this paper, elaborating on the basis of some formal analogy, recently laid bare by the authors, between possibility theory and formal concept analysis, we suggest other bridges between theories for which the concept of granulation is central. We highlight the common features between the notion of extensional fuzzy set with respect to a similarity relation and the notion of formal concept. We also discuss the case of fuzzy rough sets. Thus, we point out some fruitful cross-fertilizations between the possibilistic representation of information and several views of granulation emphasizing the idea of clusters of points that can be identified respectively on the basis of their closeness, or of their common labeling in terms of properties.
\end{abstract}

Keywords Possibility theory - Formal concept analysis . Extensional fuzzy set · Rough set · Granulation

\section{Introduction}

The issue of how to describe items is at the basis of any representation framework and naturally involves notions of similarity and uncertainty. Similarity is instrumental for grouping items having close or common features on the one hand. On the other hand, there is a need for coping with the fact that information may be incomplete or not precise

Didier Dubois

didier.dubois@irit.fr

1 IRIT, CNRS \& Université de Toulouse, Toulouse, France enough, which is a source of uncertainty. In the nonBoolean setting these notions can be couched in the setting of fuzzy sets (Zadeh 1965). It has been already emphasized in Dubois and Prade (1997) that fuzzy set membership functions can be interpreted diversely, in terms of similarity (Bellman et al. 1966; Zadeh 1971), uncertainty (Zadeh 1978, 2005) and even preferences (Bellman and Zadeh 1970). These different views have generally led to distinct families of important developments in data analysis and learning, in approximate reasoning, and in decision making, respectively.

The idea of granulation is at the heart of any knowledge representation system, as it points out that mathematical universes of discourse must be partitioned in agreement with the limitations of human perception. Generally we work with more or less well-defined partitions of idealized measurement scales; for instance the real line is too refined for human limited perception of closeness. Zadeh (1997) has emphasized the importance of granulation and granular computing and the need to cast them in a non-Boolean setting, introducing the idea of a fuzzy granules: indeed indistinguishability between two quantities gradually takes place when they get closer to each other, so that the threshold under which they become indistinguishable is fuzzy. Moreover, he makes it clear that uncertainty due to granular descriptions is possibilistic rather than probabilistic, generally.

This discussion paper intends to illustrate the idea that some links can be established at the theoretical level between different concerns related to granular computing, on the basis of formal analogies that can be laid bare between the corresponding formal settings. In the following, we successively consider four settings: possibility theory (Zadeh 1978; Dubois and Prade 1988), formal concept analysis (FCA) (Ganter and Wille 1999), 
extensional fuzzy sets (Höhle 1988) and rough sets (Pawlak 1991).

The first one, possibility theory, aims at providing a representation setting for epistemic uncertainty where partial ignorance can be encoded, and where a distinction can be made between what is somewhat certain and what is just possible to some extent. Possibility theory uses maximum and minimum operations rather than addition and product like probability theory and involves 4 set functions according to whether, for each event, one focuses on the maximum possibility value reflecting the event or its opposite, or yet the minimum possibility value.

The other three settings, sometimes apparently very different and developed completely independently, are concerned with the ideas of grouping items either because they can be gathered under the umbrella of the same formal concept, or because they are geometrically close enough to constitute fuzzy singletons, or yet because they share the same description in a database. The connection between extensional fuzzy sets and FCA was already discussed by Bělohlávek (2002), and the connection between extensional fuzzy sets and fuzzy rough sets was noticed by Boixader et al. (2000) (see also the monograph by Recassens (2010)).

Here, we first illustrate the interest of the parallel between possibility theory and formal concept analysis that we initiated in Dubois et al. (2007) and further developed in Djouadi et al. (2010), Dubois and Prade (2012). We recall the links between FCA and the formalism of rough sets in the special case of equivalence relations. Then, we indicate that this worth-noticing parallel carries over to the theory of extensional fuzzy sets and fuzzy rough sets, relying on previous technical studies. Interestingly enough, such links echo concerns often expressed by Zadeh in the last decade about the need for developing the ideas of granulation and granular computing in the setting of fuzzy sets (Zadeh 1997). The aim of this position paper is to encourage cooperation between schools of research that handle similar notions in various fields around the idea of granular computing.

The paper is organized as follows. Section 2 considers possibility theory and formal concept analysis in the crisp case. It shows that the four set functions naturally associated with the possibility theory setting have counterparts in the formal concept analysis framework. The benefit of introducing more operators in the latter theory is exemplified by recalling a connection, not considered in standard formal concept analysis, which granulates a formal context into independent formal sub-contexts. Finally, the bridge with rough sets is obtained by restricting to relations between objects, and it shows the formal analogy between concepts and clusters, independent subcontexts and granules. Then, Sect. 3 considers the non-Boolean case. It recalls the theory of extensional fuzzy sets and the representation of fuzzy extensions of equivalence relations. Then it parallels two views of granulation, namely the one at work in formal concept analysis and the one underlying the theory of extensional fuzzy sets. Finally, we bring fuzzy rough sets into the picture.

\section{From formal concept analysis to possibility theory}

Formal concept analysis associates any considered object with the set of its properties, via a formal context modeled by a binary relation $R$, a subset of the Cartesian product of the set of objects $\mathcal{O}$ and the set of properties $\mathcal{P}$.

An object is denoted by $x$, or $x_{i}$ in case we consider several ones at the same time. It is interesting to notice that in fact, an object may either refer to a particular, unique item, or to a generic item representative of a class of items sharing the same description. A subset of objects will be denoted by a capital letter $X$, and we shall write $X=\left\{x_{1}, \ldots, x_{i}, \ldots, x_{m}\right\}$. A set of objects associated with their respective sets of properties defines a formal context $R \subseteq \mathcal{O} \times \mathcal{P}$ (Ganter and Wille 1999). An object $x$ is associated with its description, denoted by $\partial(x)$. In the following, we only consider simple descriptions, expressible in terms of a subset $Y$ of properties $y_{j}$, namely, $Y=\left\{y_{1}, \ldots, y_{j}, \ldots, y_{n}\right\}$. Let $R(x)=\{y \in \mathcal{P} \mid(x, y) \in R\}$ be the set of properties of object $x$, and $R^{-1}(y)=\{x \in$ $\mathcal{O} \mid(x, y) \in R\}$ is the set of objects having property $y$. In such a case, we shall write $\partial(x)=R(x)=Y$.

The classical setting of formal concept analysis defined from a formal context relies on a single operator $R^{\triangle}$ that associates a subset of objects with the set of properties they share.

$R^{\triangleright}(X)=\left\{y \in \mathcal{P} \mid R^{-1}(y) \supseteq X\right\}=\cap_{x \in X} R(x)$.

$R^{\triangle}(X)$ is a partial conceptual characterization of objects in $X$. Objects in $X$ have all properties in $R^{\triangle}(X)$, but they may have some others (that are not shared by all objects in $X$ ). Conversely, $R^{-1 \triangle}(Y)=\{x \in \mathcal{O} \mid R(x) \supseteq Y\}=\cap_{y \in Y} R^{-1}(y)$ is the set $X$ of objects having all properties in $Y$.

In the setting of FCA, a formal concept (Ganter and Wille 1999) is defined as a pair $(X, Y) \in \mathcal{O} \times \mathcal{P}$ such that

$R^{\triangle}(X)=Y$ and $R^{-1 \triangle}(Y)=X$.

In this case $Y$ is also the maximal set of properties shared by all objects in $X$. It forms a Galois connection, and we have: 
Proposition 1 (Ganter and Wille 1999) The following properties of pairs $(X, Y)$ are equivalent

1. $R^{\triangle}(X)=Y$ and $R^{-1 \triangle}(Y)=X$

2. $(X, Y)$ is maximal such that $X \times Y \subseteq R$

A formal concept $(X, Y)$ is, thus, a maximal sub-rectangle in the formal context $R$. Let $R_{*}$ be the set union of all formal concepts extracted from $R$. Then $R_{*}=R$, by construction.

\subsection{Describing imprecise objects using possibility distributions}

In contrast with formal contexts, a useful kind of structured description of objects is in terms of attributes (Pawlak 1981). Let $a$, and $A=\left\{a_{1}, \ldots, a_{k}, \ldots, a_{r}\right\}$, respectively denote an attribute, and a set of attributes. The value of attribute $a$ for $x$ is denoted by $a(x)=u$, where $u$ belongs to the attribute domain $U_{a}$. In this case, we shall write $\partial(x)=\left(a_{1}(x), \ldots, a_{k}(x), \ldots, a_{r}(x)\right)=\left(u_{1}, \ldots, u_{k}, \ldots, u_{r}\right)$. This corresponds to a completely informed situation where all the considered attribute values are known for $x$. When this is not the case, the precise value $a_{k}(x)$ will be replaced by the possibility distribution $\pi_{a_{k}(x)}$. Such a possibility distribution (Zadeh 1978) is a mapping from $U_{a_{k}}$ to $[0,1]$, or more generally any linearly ordered scale. Then $\pi_{a_{k}(x)}(u) \in[0,1]$ estimates to what extent it is possible that the value of $a_{k}$ for $x$ is $u$. 0 means impossibility; several distinct values may be fully possible (i.e., at degree 1 ). The characteristic function of an ordinary subset is a particular case of a possibility distribution. Precise information corresponds to the characteristic function of singletons.

An elementary property $y$ can then be viewed as a subset $A_{y}$ of a single attribute domain $U_{a}$, i.e., $y \subseteq U_{a}$. Note that while $Y$ is a conjunctive set of properties (for instance an object possesses all properties in $Y$ ), property $y$, is a disjunctive set $A_{y}$ of mutually exclusive values, one of which is the value of a single-valued attribute that is ill-known for some object $x$.

Four set functions in possibility theory are now recalled (Dubois and Prade 1998a), emphasizing the symmetrical roles played by the object $x$ and the attribute value $u$, a point of view unusual in possibility theory, but echoing the symmetrical role played by objects and properties in formal concept analysis. See Dubois and Prade (2015) for a more complete introduction to the use of the four set functions in possibility theory.

\subsection{Set functions in possibility theory}

Let $\pi_{a(x)}(u)$ denote the possibility that object $x$ has value $u \in U$ according to attribute $a$. For simplicity, we only consider the single-valued attribute case here (the actual value of $x$ is not a set). The function $\pi_{a(\cdot)}(\cdot)$ defines a fuzzy set over $\mathcal{O} \times U$ (objects vs. attribute domain). We assume that $\pi_{a}$ is bi-normalized: $\forall x \in \mathcal{O}, \exists u \in U, \pi_{a(x)}(u)=1$ and $\forall u \in U, \exists x \in \mathcal{O}, \pi_{a(x)}(u)=1$. This means that for any object $x$, there is some fully possible value for attribute $a$, and that for any value $u$ there is an object $x$ that takes this value. Let $X$ be a set of objects, and $y \subseteq U$ be a property. Then, one can define four set functions, each defined in two domains, respectively, the set of objects and the attribute domain:

1. Possibility measures (Zadeh 1978), denoted by $\Pi$ :

$$
\begin{aligned}
\Pi_{u}(X) & =\max _{x \in X} \pi_{a(x)}(u) \\
\Pi_{x}(y) & =\max _{u \in y} \pi_{a(x)}(u) .
\end{aligned}
$$

$\Pi_{u}(X)$ estimates to what extent it is possible that there is an object in $X$ having value $u$, while $\Pi_{x}(y)$ is the possibility that object $x$ has property $y$. Function $\Pi$ is an indicator of non-empty intersection of the fuzzy set, whose membership function is the possibility distribution, with an ordinary subset. They are measures of "potential possibility". Clearly, $\Pi$ is max-decomposable with respect to set union.

2. The dual measures of necessity $N$ (or "actual necessity") (Dubois and Prade 1980):

$$
\begin{aligned}
N_{u}(X) & =\min _{x \notin X} 1-\pi_{a(x)}(u) \\
N_{x}(y) & =\min _{u \notin y} 1-\pi_{a(x)}(u) .
\end{aligned}
$$

$N_{u}(X)$ estimates to what extent it is certain (necessarily true) that all objects that have value $u$ lie in $X$, while $N_{x}(y)$ is the certainty that object $x$ has property $y$. Note that $N_{x}(y)=1-\Pi_{x}(\bar{y})$ where $\bar{y}=U \backslash y$. Function $N$ may be viewed as an indicator of inclusion of the fuzzy set whose membership function is the possibility distribution into an ordinary subset. And $N$ is mindecomposable with respect to set intersection.

3. The measures of "actual (or guaranteed) possibility" (Dubois and Prade 1992)

$$
\begin{aligned}
\Delta_{u}(X) & =\min _{x \in X} \pi_{a(x)}(u) \\
\Delta_{x}(y) & =\min _{u \in y} \pi_{a(x)}(u)
\end{aligned}
$$

$\Delta_{u}(X)$ estimates to what extent it is possible that all objects in $X$ have value $u$, while $\Delta_{x}(y)$ estimates the possibility that object $x$ may take any value in $y . \Delta$ may be viewed as a degree of inclusion of an ordinary subset into the fuzzy set whose membership function is the possibility distribution. $\Delta$ is min-decomposable with respect to set union. 
4. The dual measures of "potential necessity or certainty" (Dubois and Prade 1992)

$$
\begin{gathered}
\nabla_{u}(X)=1-\min _{x \notin X} \pi_{a(x)}(u) \\
\nabla_{x}(y)=1-\min _{u \notin y} \pi_{a(x)}(u)
\end{gathered}
$$

$\nabla_{u}(X)$ estimates to what extent there exists at least one object outside $X$ that has a low degree of possibility of having value $u$, while $\nabla_{x}(y)$ is the degree to which there is an impossible value for $a(x)$ outside $y$. Note that $\nabla_{x}(y)=1-\Delta_{x}(\bar{y}) . \nabla$ is an indicator of non-full coverage of the considered universe by the fuzzy set whose membership function is the possibility distribution together with an ordinary subset. $\nabla$ is maxdecomposable with respect to set intersection.

\subsection{Application to the formal context setting}

In Dubois et al. (2007), the setting of formal concept analysis has been enlarged with the introduction of three other operators. We now recall these four operators. They are counterpart, in the setting of a formal context, of the above set functions from possibility theory.

Namely, let $R$ be the formal context (viewed as a Boolean table). Then knowing only that an object $x$ has some property $y$, the set $R^{-1}(y)=\{x \in \mathcal{O} \mid(x, y) \in R\}$ is the set of possible objects pointed out by the elementary piece of knowledge "the object has property $y$ " (in the context $R$ ). This suggests a possibilistic reading of formal concept analysis: a formal counterpart of possibility theory set functions can be laid bare in this framework.Then, four remarkable sets can be associated with a subset $X$ of objects (the notations have been chosen here in order to emphasize the parallel with possibility theory) (Dubois et al. 2007; Dubois and Prade 2009):

- $\quad$ The set $R^{\Pi}(X)$ of properties that are possessed by at least one object in $X$ :

$R^{\Pi}(X)=\left\{y \in \mathcal{P} \mid R^{-1}(y) \cap X \neq \emptyset\right\}=\cup_{x \in X} R(x)$.

Clearly, we have $R^{\Pi}\left(X_{1} \cup X_{2}\right)=R^{\Pi}\left(X_{1}\right) \cup R^{\Pi}\left(X_{2}\right)$.

- The set $R^{N}(X)$ of properties s. t. any object that satisfies one of them is necessarily in $X$ :

$R^{N}(X)=\left\{y \in \mathcal{P} \mid R^{-1}(y) \subseteq X\right\}=\cap_{x \notin X} \overline{R(x)}$,

where the overbar denotes complementation. In other words, possessing any property in $R^{N}(X)$ is a sufficient condition for belonging to $X$. Moreover, we have $R^{N}\left(X_{1} \cap X_{2}\right)=R^{N}\left(X_{1}\right) \cap R^{N}\left(X_{2}\right)$ and $R^{N}(X)=\overline{\left.R^{\Pi} \bar{X}\right)}=$ $\mathcal{P} \backslash R^{\Pi}(\bar{X})$.

- $\quad$ The set $R^{\triangle}(X)$ of properties shared by all objects in $X$ :

$$
R^{\triangleright}(X)=\left\{y \in \mathcal{P} \mid R^{-1}(y) \supseteq X\right\}=\cap_{x \in X} R(x) .
$$

In other words, satisfying all properties in $R^{\triangle}(X)$ is a necessary condition for an object to belong to $X$. Clearly, $R^{\triangle}\left(X_{1} \cup X_{2}\right)=R^{\triangle}\left(X_{1}\right) \cap R^{\triangle}\left(X_{2}\right)$.

- The set $R^{\nabla}(X)$ of properties that are not satisfied by at least one object in $\bar{X}$.

$$
R^{\nabla}(X)=\left\{y \in \mathcal{P} \mid R^{-1}(y) \cup X \neq \mathcal{O}\right\}=\cup_{x \notin X} \overline{R(x)} .
$$

Note that $R \nabla(X)=\overline{R^{\triangle}(\bar{X})}=\mathcal{P} \backslash R^{\triangle}(\bar{X})$. In other words, in context $R$, for any property in $R \nabla(X)$, there exists at least one object outside $X$ that misses it. Moreover, we have $R^{\nabla}\left(X_{1} \cap X_{2}\right)=R^{\nabla}\left(X_{1}\right) \cup R^{\nabla}\left(X_{2}\right)$.

A number of remarks are worth noticing:

- In negative similarity to $R^{\triangle}(X), \overline{R^{\Pi}(X)}$ provides a negative conceptual characterization of objects in $X$ since it gathers all the properties that are never satisfied by any object in $X$.

- $R^{N}(X) \cap R^{\triangle}(X)$ is the set of properties possessed by all objects in $X$ and only by them.

- $R^{\Pi}(X)$ and $R^{N}(X)$ are isonotonic (they become larger when $X$ increases), while $R^{\triangle}(X)$ and $R^{\nabla}(X)$ are antitonic (they become smaller when $X$ increases).

The four subsets $R^{\Pi}(X), R^{N}(X), R^{\triangle}(X)$, and $R \nabla(X)$ have been considered (with different notations) without any mention of possibility theory by different authors. The standard operator in FCA is $R^{\triangle}$. Düntsch et al. (1999, 2003) calls $R^{\triangle}$ a sufficiency operator, and its representation capabilities are studied in the theory of Boolean algebras. Taking inspiration as the previous authors from rough sets Pawlak (1991), Yao (2004, 2006) also consider these four subsets. In both cases, the four operators were introduced. See also (Popescu 2004; Georgescu and Popescu 2004). The interest of the bridge between possibility theory and FCA is that it enables a systematic investigation of alternative connections between objects and properties to be carried out; they differ from the standard Galois connection of FCA.

\subsection{Application to formal context decomposition}

It can be checked that $R^{\nabla}$ defines the same Galois connection as the one defined from $R^{\triangle}$, while $R^{N}$ (or equivalently $R^{\Pi}$ ) induces another kind of connection, which is now described.

The connection defined from $R^{N}$ proceeds in a similar formal way as when defining formal concepts (Dubois and Prade 2009; Djouadi et al. 2010). Namely, let us consider pairs $(X, Y)$ s.t. $R^{N}(X)=Y$ and $R^{-1 N}(Y)=X$. We can show these pairs also satisfy $R^{\Pi}(X)=Y$ and $R^{-1 \Pi}(Y)=X$. 
Moreover, the pairs $(X, Y)$ s.t. $R^{N}(X)=Y$ and $R^{-1 N}(Y)=$ $X$ allow us to characterize independent sub-contexts (i.e., that have no common objects and no common properties), and are thus of interest for the decomposition of a formal context into smaller independent ones. These results are expressed through the following:

Proposition 2 (Dubois and Prade 2012) The following properties of pairs $(X, Y)$ are equivalent

1. $R^{N}(X)=Y$ and $R^{-1 N}(Y)=X$

2. $R^{N}(\bar{X})=\bar{Y}$ and $R^{-1 N}(\bar{Y})=\bar{X}$

3. $R^{\Pi}(X)=Y$ and $R^{-1 \Pi}(Y)=X$

4. $R \subseteq(X \times Y) \cup(\bar{X} \times \bar{Y})$

Thus, $(X, Y)$ and $(\bar{X}, \bar{Y})$ are two independent sub-contexts in $R$, in the sense that there is no object/property pair $(x, y)$ from context $R$ in $X \times \bar{Y}$ nor in $\bar{X} \times Y$. There is no minimality requirement in the inclusion property 4 of the above proposition. In particular, the pair $(\mathcal{O}, \mathcal{P})$ trivially satisfies it. However, this result leads to a decomposition of $R$ into a disjoint union of minimal independent sub-contexts. Indeed, suppose two pairs $\left(X_{1}, Y_{1}\right),\left(X_{2}, Y_{2}\right)$ satisfy the above proposition. It implies that for instance, the pair $\left(X_{1} \cap X_{2}, Y_{1} \cap Y_{2}\right)$ satisfies it (it can be checked that $\left.R^{N}\left(X_{1} \cap X_{2}\right)=Y_{1} \cap Y_{2}\right)$, and likewise with any element of the partition refining both partitions $\left(X_{1}, \overline{X_{1}}\right)$ and $\left(X_{2}, \overline{X_{2}}\right)$. Due to point 4 of the proposition, it yields

$$
R \subseteq\left(\left(X_{1} \times Y_{1}\right) \cup\left(\overline{X_{1}} \times \overline{Y_{1}}\right)\right) \cap\left(\left(X_{2} \times Y_{2}\right) \cup\left(\overline{X_{2}} \times \overline{Y_{2}}\right)\right),
$$

where the intersection on the right-hand side comes down to the union of subcontexts $\left(X_{1} \cap X_{2}\right) \times\left(Y_{1} \cap Y_{2}\right)$, $\left(X_{1} \cap \overline{X_{2}}\right) \times\left(Y_{1} \cap \overline{Y_{2}}\right),\left(\overline{X_{1}} \cap X_{2}\right) \times\left(\overline{Y_{1}} \cap Y_{2}\right),\left(\overline{X_{1}} \cap \overline{X_{2}}\right) \times$ $\left(\overline{Y_{1}} \cap \overline{Y_{2}}\right)$. The decomposition of $R$ into minimal subcontexts is achieved by taking the following intersection (Dubois and Prade 2012)

$$
R^{*}=\bigcap_{(X, Y): R^{N}(X)=Y, R^{-1 N}(Y)=X}(X \times Y) \cup(\bar{X} \times \bar{Y}) .
$$

In general, $R \subset R^{*}$.

Example (Dubois and Prade 2009). Figure 1 presents a formal context. Pairs $(\{6,7,8\},\{c, d, e\}),(\{5,6,7,8\}$, $\{d, e\}),(\{2,3,4\},\{g, h\})$ are examples of formal concepts, while pairs $(\{5,6,7,8\},\{a, b, c, d, e\}),(\{2,3,4\}$, $(\{f, g, h\}),(\{1\},\{i\})$ are minimal subcontexts. And it can be checked that

$R \subset\{5,6,7,8\} \times\{a, b, c, d, e\} \cup\{2,3,4\} \times\{f, g, h\} \cup\{1\} \times\{i\}$.

The connection $\left(R^{\Pi}, R^{-1 \Pi}\right)$ has been originally introduced by Georgescu and Popescu (2004) and studied in the framework of multivalued data tables with entries in a

\begin{tabular}{l|c|cccccccc}
\multicolumn{10}{c}{ objects } \\
\hline $\mathrm{p}$ & & 1 & 2 & 3 & 4 & 5 & 6 & 7 & 8 \\
\hline $\mathrm{r}$ & $a$ & & & & & & & $\times$ & \\
$\mathrm{O}$ & $b$ & & & & & $\times$ & $\times$ & & \\
$\mathrm{p}$ & $c$ & & & & & & $\times$ & $\times$ & $\times$ \\
$\mathrm{e}$ & $d$ & & & & & $\times$ & $\times$ & $\times$ & $\times$ \\
$\mathrm{r}$ & $e$ & & & & & $\times$ & $\times$ & $\times$ & $\times$ \\
$\mathrm{t}$ & $f$ & & $\times$ & & $\times$ & & & & \\
$\mathrm{i}$ & $g$ & & $\times$ & $\times$ & $\times$ & & & & \\
$\mathrm{e}$ & $h$ & & $\times$ & $\times$ & $\times$ & & & & \\
$\mathrm{s}$ & $i$ & $\times$ & & & & & & & \\
\hline
\end{tabular}

Fig. 1 Formal concepts and sub-contexts

residuated lattice, but its practical significance for Boolean data tables was not really discussed. These authors call a pair of operators $(f, g)$, where $f: 2^{\text {Obj }} \rightarrow 2^{\text {Prop }}, g: 2^{\text {Prop }} \rightarrow$ $2^{\mathrm{Obj}}$ relating the subsets of objects and properties, a conjugated pair of operators if and only if

$$
X \cap g(Y)=\emptyset \Longleftrightarrow f(X) \cap Y=\emptyset .
$$

It is easy to see that $\left(R^{\Pi}, R^{-1 \Pi}\right)$ is a conjugated pair of operators. To see it note that $R^{\Pi}(X) \cap Y=\emptyset$ also writes $\cup_{x \in X}(R(x) \cap Y)=\emptyset$. It holds if and only if $R \cap(X \times Y)=\emptyset$. So, by symmetry, it is equivalent to $R^{-1 \Pi}(Y) \cap X=\emptyset$.

In terms of the dual operator $N$, the conjugation property reads $Y \subseteq R^{N}(\bar{X}) \Longleftrightarrow X \subseteq R^{-1 N}(\bar{Y})$. However, this connection is not a Galois connection. One reason is that iterating $R^{N}$ and $R^{-1 N}$ does not yield an idempotent operation. Of course the same holds for $R^{-1 \Pi}\left(R^{\Pi}(X)\right)$, $R^{\Pi}\left(R^{-1 \Pi}(Y)\right), R^{N}\left(R^{-1 N}(Y)\right)$. For instance, on the data table of Fig. $1, R^{-1 N}(\{a, c, d, e\})=\{7,8\}, R^{N}(\{7,8\})=$ $\{a\}$ and $R^{-1 N}(\{a\})=\emptyset$.

Through the notions of formal sub-contexts and of formal concepts, one sees two aspects of granulation at work. Namely, on the one hand independent sub-contexts are separated granules, while inside each sub-context, formal concepts $(X, Y)$ are identified where each object in $X$ is associated with each property in $Y$, which can be viewed as a cluster. Note that in the special case when a formal context can be decomposed into independent formal concepts (i.e., each minimal sub-context is a formal concept), we have a perfect granulation: two objects are either identical in terms of properties, or they do not have any property in common. However, in the general case, objects in the extension of a formal concept may not be fully similar since they may also possess properties outside the intension of the concept. They are only similar with respect to the properties associated to 
the formal concept. In practice, it may be interesting to introduce some tolerance in the definition of formal subcontexts and concepts (Dubois and Prade 2012; Gaume et al. 2010), leading to a more permissive and approximate view of granules or clusters.

Besides, the above results can be also expressed in terms of bipartite graph clustering, where

- There are two kinds of nodes corresponding to objects and properties.

- Formal concepts correspond to sets of object nodes connected to all nodes in subsets of property nodes.

- The decomposition into independent subcontexts corresponds to connected components of the bipartite graph (each node of one set being related to at least one node of another set of the opposite type).

One can then take advantage of this exact parallel between formal concept analysis and bipartite graph analysis (Gaume et al. 2010).

\subsection{From formal concept analysis to rough sets}

The concept of granulation is even more central in rough set theory (Pawlak 1991). Rough set theory focuses on the impossibility to precisely describe any set of objects when the properties used to describe them are not enough discriminant. One connection between FCA and rough sets is that the latter also start from a data table like a formal context (we assume Boolean attributes in the following). Let $X_{y}$ be the set of objects satisfying the property $y$. Then there exists a partition generated on $\mathcal{O}$ by the family of subsets $\left\{X_{y}: y \in \mathcal{P}\right\}$, each element of which is an interpretation of the propositional language induced by properties in $\mathcal{P}$, i.e. it is of the form $\times_{y \in \mathcal{P}} X_{y}^{e_{y}}, e_{y} \in\{-1,1\}$, with $X_{y}^{e_{y}}=X_{y}$ if $e_{y}=1$, and $X_{y}^{e_{y}}=\bar{X}_{y}$ if $e_{y}=-1$. If $R$ is the formal context, then two objects $x$ and $x^{\prime}$ are said to be indiscernible (they are in the same element of the partition) if they share the same properties (which writes $R(x)=R\left(x^{\prime}\right)$ ). It enables the data table to be reduced to the case where no two lines in $R$ are equal.

The rough set approach considers the above partition of the universe $\mathcal{O}$ of objects, say $X_{1}, \ldots, X_{k}$ induced by the properties via the equivalence relation $E$ defined by $E\left(x, x^{\prime}\right)=1$ if and only if $R(x)=R\left(x^{\prime}\right)$ and 0 otherwise. So, all that is known about any object in $\mathcal{O}$ is which subset of the partition it belongs to. So each subset $X$ of objects is only known in terms of its upper and lower approximations, a pair $\left(X_{*}, X^{*}\right)$ such that

$X^{*}=\bigcup\left\{X_{i}, X_{i} \cap X \neq \emptyset\right\}$ and $X_{*}=\bigcup\left\{X_{i}, X_{i} \subseteq X\right\}$.

It is clear that $(A \cap B)^{*} \subseteq A^{*} \cap B^{*}$ and $A_{*} \cup B_{*} \subseteq(A \cup B)_{*}$. Note that an equivalence class of relation $E$ corresponds to a specialization of both a formal concept and a formal independent subcontext.

To summarize the links between rough sets and FCA, a formal concept can be viewed as a 2-dimensional extension of an equivalence class. A formal context is a 2-dimensional extension of equivalence relation if it can be decomposed into a disjoint union of elementary sub-contexts, each of which forms a single formal concept. In that case, the context we start with is the perfect extension of the equivalence relation to the 2-dimensional setting.

Another way of putting together FCA and rough sets consist in putting both on a cube of oppositions, whereby their connections to possibility theory functions can be highlighted; see Ciucci et al. (2014).

\subsection{Clusters and granules}

Assume now a general relation $S$ between objects, that is $S \subseteq \mathcal{O} \times \mathcal{O}$. It can be viewed as a directed graph whose nodes form the set $\mathcal{O}$. We assume the relation is serial, that is $\forall x \in \mathcal{O}, S(x) \neq \emptyset$, and its converse $S^{-1}$ is serial too; we say that $S$ is biserial. The definition of a formal concept then is a maximal Cartesian product $A \times B \subseteq \mathcal{O} \times \mathcal{O}$ contained in $S$. We can still define it as satisfying the two equalities $S^{\Delta}(A)=B$ and $S^{-1 \Delta}(B)=A$. Suppose the relation $S$ is symmetrical, in order to capture some idea of proximity. Then, the maximal Cartesian products $A \times B$ contained in $S$ are of the form $C \times C \subseteq S$, i.e., they are maximal cliques in the non-directed graph associated to $S$ : the two equalities defining formal concepts then boil down to a single one:

$S^{\triangle}(C)=\cap_{x \in C} S(x)=C$,

which expresses the fact that each node in $C$ is related to all nodes in $C$, and corresponds to one major feature of a cluster. We call the set $C$ a tight cluster, because each element in $C$ is close to all other elements in $C$. Note that $S$ must be reflexive (an element is close to itself), otherwise there is no such tight cluster. Then it is enough to require that $C \subseteq S^{\triangle}(C)$ since the other inclusion trivially holds.

Alternatively we can consider minimal Cartesian products $A \times B$ such that $S \subseteq(A \times B) \cup(\bar{A} \times \bar{B})$, which satisfy the two equalities $S^{\Pi}(A)=B$ and $S^{-1 \Pi}(B)=A$. If the relation $S$ is symmetrical, it corresponds to the minimal Cartesian products $B \times B$ such that $S \subseteq(B \times B) \cup(\bar{B} \times \bar{B})$. They satisfy the equality

$S^{\Pi}(B)=\cup_{x \in B} S(x)=B$,

This is because the identity (7) is equivalent to

$S \subseteq(B \times B) \cup(\bar{B} \times \bar{B})$.

If $S$ is reflexive, it is enough to require that $S^{\Pi}(A) \subseteq A$ instead of (7) since the other inclusion trivially holds. 
Minimal subsets $G$ that satisfy (8) are such that each element of $G$ is related to at least one element of $B$ and to none outside $G$. This is the other expected property of a cluster, but we can call it a loose granule. Loose granules of $S$ form the set $\mathcal{G}(S)$ and correspond to maximal connected components in the non-directed graph associated to $S$. Note that tight clusters can only be found inside loose granules: for any tight cluster $A$, there exists a loose granule containing it. Tight clusters and loose granules cannot be told apart if the relation $S$ is moreover transitive.

Proposition 3 Consider a symmetric serial relation $S$. Then $S=E$ is an equivalence relation if and only if its loose granules and tight clusters coincide.

Proof If $S=E$ is an equivalence relation, it is easy to check that loose granules and tight clusters coincide. Conversely, if loose granules and tight clusters in $S$ coincide, then an element in a loose granule is connected to all elements in this granule and to none outside. So $S$ corresponds to a partition, and is an equivalence relation.

It is also clear that the relation $S^{*}=\cap_{G \in \mathcal{G}(S)}(G \times G) \cup$ $(\bar{G} \times \bar{G})$ is transitive, and is actually the transitive closure $\operatorname{cl}(S)$ of $S$. As the transitive closure of $S$ is reflexive, it is thus be an equivalence relation. So loose granules form a partition of $\mathcal{O}$. More precisely:

Proposition 4 Consider a symmetric serial relation $S$. The tight clusters of $c l(S)$ are the loose granules of $S$.

Proof Let $B$ be a loose granule of $S$. Since the graph with nodes in $B$ is connected, all nodes in $B$ will be related to all nodes in $B$ in the graph of the transitive closure of $S$, but not to any node outside $B$. Hence $B$ is a tight cluster of $c l(S)$. If $B$ is not contained in a loose granule of $S$, then it is made of more than one connected component, hence they remain disconnected via transitive closure. So, $B$ will not be a loose granule of $c l(S)$, a fortiori not a tight one.

So it can be seen that a reflexive and symmetric relation represents a partition of separated loose granules, each possibly containing several tight clusters (that may overlap), which makes it very similar to a formal context.

\section{Extensional fuzzy sets and fuzzy contexts}

The concept of extensional fuzzy set with respect to a fuzzy equality, proposed in Höhle (1988), Valverde (1985), further developed by Boixader et al. (2000), Klawonn (2000), and Recassens (2010) also embeds ideas of granulation. It is a multivalued extension of the decomposition of a relation into tight clusters and loose granules recalled above. This approach has mathematical roots in category theory and Heyting algebras (Higgs 1973), whereby a multivalued notion of equality is used. As we are going to see, although defined in a different algebraic setting and on the basis of a completely different intuition, it is also closely related to the gradual version of formal concept analysis (Bělohlávek 1999, 2002; Popescu 2004; Georgescu and Popescu 2004).

\subsection{Fuzzy singletons and extensional hulls}

Let $E$ be a fuzzy similarity relation defined on a universe $U$. For simplicity, we assume the use of the scale $[0,1] . E$ is supposed to be

- $\quad$ reflexive $(E(u, u)=1)$,

- $\operatorname{symmetric}(E(u, v)=E(v, u))$,

- $\quad *$-transitive $(E(u, v) * E(v, w) \leq E(u, w))$,

where $*$ is a triangular norm (Klement et al. 2000) (i.e., $*$ is increasing in the broad sense, associative, commutative and such that $0 * 0=0,1 * a=a$ ). It was first proposed by Zadeh (1971) when $*=\min$.

Such a fuzzy relation models a form of proximity between elements of the set $U$. Relation $E$ is sometimes also called "fuzzy equivalence" (Boixader et al. 2000), "(fuzzy) equality relation" (Klawonn 2000), or "(fuzzy) indistinguishability relation" (Valverde 1985), or yet "indiscernibility relation" (Pawlak 1991). Note that the terms "indistinguishability" and "equality" refer to quite different intuitions, only the former being naturally understood as the weak version of an equivalence relation (Dubois and Prade 1998b). Indeed, one may argue that the 1-cut of a fuzzy equality should be the standard equality (i.e. $E(u, v) \neq 1$ if $u \neq v)$, i.e., separability holds. On the contrary, the name indistinguishability relation is denying separability. In the following, we do not require separability.

Interesting choices for operation $*$ are min, product or the Łukasiewicz t-norm $a *_{\mathrm{t}} b=\max (0, a+b-1)$. Fuzzy similarity relation are the negative of distances or metrics (Boixader et al. 2000; Recassens 2010). The min-transitivity makes a fuzzy similarity closely related to an ultrametric. The $*_{\mathrm{E}}$-transitivity corresponds to the triangular inequality.

A fuzzy set $F$ is said to be extensional with respect to $E$ (Höhle 1988; Klawonn 2000) iff

$\forall u, v, F(u) * E(u, v) \leq F(v)$

Let $F \circ E$ be obtained as $F \circ E(v)=\max _{u \in U} F(u) * E(u, v)$. It is clear that due to the properties of $E$, it always holds that $F \subseteq F \circ E$. Moreover $F \circ E$ can be written as $E^{\Pi}(F)$ as it is the fuzzy set contains all elements in the vicinity of $F$. So, Eq. (9) can be written as $F \circ E(v)=F$. Equation (9) generalizes the condition $S^{\Pi}(B)=B$ in Eq. (7), so that we can also write it as $E^{\Pi}(B)=B$. 
Consider now the implication connective $\rightarrow$ associated to $*$ by residuation, i.e., we assume $a * b \leq c \Leftrightarrow a \leq b \rightarrow c$. The extensionality of $F$ is obviously equivalent to

$\forall u, v, F(u) \leftrightarrow F(v) \geq E(u, v)$

where $a \leftrightarrow b=\min (a \rightarrow b, b \rightarrow a)$, using residuation and the symmetry of $E$. Equation (10) generalizes the property (8) $S \subseteq(B \times B) \cup(\bar{B} \times \bar{B})$ to multivalued relations.

The extensional hull $\hat{F}$ of a fuzzy set $F$ (w.r.t. $E$ ) is then defined as

$\hat{F}=\inf \{G \mid F \subseteq G$ and $G$ is extensional w.r.t. $E\}$.

It is obvious that $F \circ E$ is extensional $\left(E^{\Pi}(F \circ E)=\right.$ $(F \circ E) \circ E=F \circ(E \circ E)=F \circ E$, since $E$ is $*$-transitive $)$ and is the extensional hull of $F$.

An important example of extensional fuzzy set is obtained by considering an element $u$ and the fuzzy set $F_{u}$ of elements similar to it, that is $F_{u}(v)=E(u, v)$ (it is a line of matrix $E$ ). $F_{u}$ is clearly the extensional hull of the singleton $\{u\}$. Note that $F_{v}(u)=F_{u}(v)$, and that if $F_{v}(u)=1$ then $F_{v}=F_{u} . F_{u}$ is the fuzzy counterpart of an equivalence class. Klawonn (2000) calls it a "fuzzy point", understood as the largest cluster of indiscernible entities around $u$, as per the fuzzy similarity relation $E$.

Each fuzzy set $F_{v}$ can be seen as a fuzzy loose granule. It is an atomic entity inside $U$ that cannot be split, if an observer whose myopic eyesight is modeled by the fuzzy similarity $E$. If $E$ is an equivalence relation (for instance, the 1-cut of a fuzzy similarity is clearly an equivalence relation), $F_{u}$ is just the equivalence class of $u$. The extensional hull of a crisp subset $A \subseteq U$ is the union of extensional hulls of all elements in the set:

$\mu_{\hat{A}}(u)=\sup _{v \in A} E(u, v)$

An interesting question whether any extensional fuzzy set takes this form. An extensional fuzzy set would then always consist of the fuzzy union of fuzzy extensional hulls of singletons, as in the crisp case. It would hold if an extensional fuzzy set coincides with the extensional hull of its core. But the latter property is not true. For instance, consider a fuzzy set $F$ containing strictly $F_{u}$ but with the same core $A$. Clearly, its extensional hull $E^{\Pi}(F)$ strictly contains $F_{u}$ but also has the same core $A$ (an equivalence class of the 1-cut of $E$ ). Hence it is not of the form $\cup_{u \in A} F_{u}$.

Höhle and Klawonn call a fuzzy singleton $F$ (w.r.t. $E$ ) a non-empty fuzzy set (i.e., $\max _{u} F(u)=1$ ) such that

$F(u) * F(v) \leq E(u, v)$

In particular we equivalently have $F(u) \leq F(v) \rightarrow E(u, v)$, $\forall v \in U$, that is,

$$
F(u) \leq \min _{v \in U} F(v) \rightarrow E(u, v) .
$$

Considering maximal fuzzy singletons, we generalize the FCA operator: they are such that $F=E^{\Delta}(F)$, since the composition on the right-hand side of the above inequality extends operation $\Delta$. Clearly, the union of two such fuzzy singletons is not a fuzzy singleton. In fact, a fuzzy singleton is a greatest fuzzy set satisfying (12). Maximal fuzzy singletons are the multivalued version of the notion of tight cluster, i.e., the specialization of a formal concept to relations over a set. ${ }^{1}$

Using a $*$-transitive similarity relation we can prove that extensional hulls of singletons are maximal fuzzy singletons.

Proposition 5 If $E$ is a *-transitive similarity relation, and $w \in U$ a singleton, then $F_{w}(u) * F_{w}(v) \leq E(u, v)$.

Proof Note that letting $F=F_{w}$ in (12), we again get the expression of the transitivity of $E$. Hence $F_{w}$ satisfies (12).

What this result shows is that fuzzy versions of tight clusters and loose granules in the sense of a fuzzy similarity relation coincide with equivalence classes $F_{u}$, just like in the classical case for equivalence relations. Due to *-transitivity, it holds that $E^{\Delta}\left(F_{u}\right)=E^{\Pi}\left(F_{u}\right)=F_{u}$, $\forall u \in U$. One question to be solved is whether there are other fuzzy sets that are at the same time extensional and are fuzzy singletons, that is whether $E^{\Delta}(F)=E^{\Pi}(F)=F$ implies that $F$ is just the extensional hull of a singleton (a fuzzy similarity class). Note that extensional hulls of crisp subsets other than singletons do not qualify as candidates as $E^{\Delta}(A)=\cap_{u \in A} E^{\Delta}(\{u\})$ and $E^{\Pi}(A)=\cup_{u \in A} E^{\Pi}(\{u\})$.

Valverde (1985) (see also Boixader et al. 2000; Klawonn 2000; Recassens 2010) considers the converse problem of generating a fuzzy relation from a family of subsets. Given a family $\mathcal{F}$ of fuzzy sets $F$ the coarsest equivalence relation $E^{\mathcal{F}}$ such that all fuzzy sets $F \in \mathcal{F}$ are extensional is

$$
E^{\mathcal{F}}(u, v)=\bigwedge_{F \in \mathcal{F}} F(u) \leftrightarrow F(v) .
$$

In the crisp case, take $\mathcal{F}$ as $\left\{A_{i}: y_{i} \in \mathcal{P}\right\}$. Then it simply says that two elements are related if and only if they belong to the same sets $A_{i}$ (they share the same properties). This equation is extended to the case where the properties are more or less important by Bělohlávek (2002).

While the coarsest fuzzy similarity relation $E^{\mathcal{F}}$ such that all fuzzy sets $F \in \mathcal{F}$ are extensional is provided above by Valverde result (13), the finest such fuzzy similarity relation $E_{\mathcal{F}}$ is of the form

\footnotetext{
${ }^{1}$ The term "singleton" here means that fuzzy singletons are atomic entities as per the indistinguishability relation $E$.
} 


$$
\begin{aligned}
E_{\mathcal{F}}(u, v) & =1 \text { if } u=v \\
& =\bigvee_{F \in \mathcal{F}} F(u) * F(v) \text { otherwise. }
\end{aligned}
$$

Moreover, Klawonn (2000) addresses the case when a collection of normalized fuzzy sets can be viewed as forming a family of fuzzy points. If $\forall F_{i} \in \mathcal{F}, \exists u_{i}$, such that $F_{i}\left(u_{i}\right)=1$, then the fact that $\mathcal{F}$ is a family of fuzzy points with respect to $E$ is equivalent to the following inequality: $\forall F_{i}, F_{j} \in \mathcal{F}$,

$$
\bigvee_{u \in U} F_{i}(u) * F_{j}(u) \leq \bigwedge_{v \in U} F_{i}(v) \leftrightarrow F_{j}(v)
$$

This condition is a fuzzy counterpart of the fact that equivalence classes (here generalized to fuzzy points) are disjoint.

\subsection{Extensional fuzzy sets and FCA: analogies}

In the Boolean case, the mathematical expressions (6) and (7) are special cases of formal concept analysis expressions. Similarly, in the multivalued case, we can generalize identities $(9,10,12)$ to the setting of FCA. First, the counterpart to (9) using a formal multivalued context is: $\forall x, y$,

$$
\begin{array}{r}
X(x) * R(x, y) \leq Y(y) \\
Y(y) * R^{-1}(y, x) \leq X(x)
\end{array}
$$

It is the multivalued version of the third point of Proposition 2 that operates a decomposition into disjoint subcontexts. It is equivalent to the counterpart of (10) and point 4 of Proposition 2 from Sect. 2.4), namely:

$\forall x, y, X(x) \leftrightarrow Y(y) \geq R(x, y)$

As already said, in the fuzzy similarity setting, there is only one Eq. (9) instead of two in FCA because the fuzzy similarity relation is symmetric. This indicates that the idea of extensional fuzzy set bears a strong analogy with the notion of formal sub-context Indeed, (16) expresses that if an object $x$ of $X$ has property $y$ then this property is in $Y$, and conversely if a property $y$ of $Y$ applies to an object $x$ then this object is in $X$, i.e., $(X, Y)$ is an independent subcontext; so an independent subcontext is extensional. Moreover, we can deal with a fuzzy extension of the notion of formal sub-context (Dubois and Prade 2009) since Eqs. (16) and (17) make sense in $[0,1]$, and not only in $\{0,1\}$.

In fact, the decomposition of $R$ into minimal contexts (forming relation $R^{*}$ in Eq. (4) above the example of Sect. 2.4) corresponds to the construction of the coarsest fuzzy similarity relation induced by a family of fuzzy sets as per Eq. (13). To see it, just consider instead of the family $\mathcal{F}$ the set of conjugated pairs obtained from the context $R$. More generally, a fuzzy relation $R$ on $U$ generates a family $\mathcal{F}(R)$ of fuzzy sets $F_{u}$ such that $F_{u}(v)=R(u, v), \forall u \in U$. Considering the coarsest fuzzy similarity relation $E^{\mathcal{F}(R)}$, it is clear that $R \subseteq E^{\mathcal{F}(R)}$ just like $R \subseteq R^{*}$ in the context decomposition framework.

Likewise, multivalued counterparts of formal concepts, as per Proposition 1 can be defined:

$X(x) * Y(y) \leq R(x, y)$,

which is equivalent to Bělohlávek $(1999,2002) \forall x, y$,

$\begin{aligned} X(x) & \rightarrow R(x, y) \geq Y(y) \\ Y(y) & \rightarrow R^{-1}(y, x) \geq X(x) .\end{aligned}$

one can see a parallel between the idea of a fuzzy point [a maximal fuzzy singleton in the sense of (12)] and the notion of formal concept. Indeed, Eq. (19) expresses that if a property $y$ is in $Y$, any object $x$ of $X$ should possess it, and conversely if an object $x$ is in $X$, any property $y$ in $Y$ should be possessed by it. And Eq. (12) of fuzzy singletons can also be expressed as $F(u) \leq E(u, v) \rightarrow F(v)$, from residuation, so that we do have that $F=E^{\Delta}(F)$ and a pair of fuzzy points $(F, F)$ is like a formal concept. So a concept $(X, Y)$ is similar to a fuzzy point. Equations (18) and (19) in fact provide a fuzzy extension of the notion of formal concept in the sense developed in Bělohlávek $(1999,2002)$, whose similarity with the extensional fuzzy set construction is thus laid bare.

It is clear that forming the union of fuzzy formal concepts in a context $R$ yields a relation $R_{*} \subseteq R$ (with equality in the crisp case). It is the counterpart of the finest fuzzy similarity relation in Eq. (14) induced by a family of fuzzy sets, while decomposing $R$ into formal contexts yields a relation $R^{*}$, defined by Eq. (4), that contains $R$, and reminds us of the coarsest relation induced by a family of fuzzy sets (13). The obvious inclusion $R_{*} \subseteq R^{*}$ is clearly the counterpart of Eq. (15).

Thus, we have exhibited a formal resemblance between two quite different views of a granulation process. There is a big difference between them, though. One is induced by an approximate equality relation, while the other is based on a binary relation defined on the Cartesian product of two different sets. In the former case, due to the properties of the fuzzy similarity relation what corresponds to concepts in FCA, and what corresponds to minimal independent subcontexts are the same (they are fuzzy points). Moreover, the fuzzy extensionality problem is to derive a fuzzy similarity relation from any family of fuzzy sets, while in FCA the issue is to find "maximal singletons" and minimal independent subrelations induced by any binary relation. However the common algebraic setting for both problems is a building block of fuzzy FCA as developed by 
Bělohlávek (2002). This algebraic setting, also used by Klawonn (2000) in his approach to extensional fuzzy sets, is the one of residuated lattices.

Lastly, the first part of expression (16) and the expression (18) are also the starting points respectively of the implication-based and of the conjunction-based views of a fuzzy rule "if $x$ is in $\tilde{X}$ then $y$ is in $\tilde{Y}$ " (Dubois and Prade 1996). Fuzzy rules defined via these two equations indeed correspond to two different ways of granulating a relation or function defined from the universe containing the (fuzzy) subset $\tilde{X}$ to the universe containing the (fuzzy) subset $\tilde{Y}$. Klawonn (2000) shows that the counterpart to inequality (15) is instrumental in the solution of fuzzy relational equations induced by the specification of fuzzy rules, especially if the fuzzy relation must be constructed using the conjunction-based view. In some sense the modeling of fuzzy rules and fuzzy formal concept analysis rely on the same basic algebraic setting and the same basic equations but have opposite programs. While fuzzy FCA tries to extract concepts from fuzzy relations modeling many-valued contexts, with a view to derive interpretable association rules, the other program is to synthesize fuzzy relations between input to output spaces from fuzzy rules expressed in natural language. The formal relations between the two areas are thus worth studying further. For instance, Bělohlávek (2009) tries to derive implicative rules from fuzzy formal contexts, using the same equation (inf $\rightarrow$ composition) as the one that turns a set of implicative rules into a fuzzy relation (Dubois and Prade 1996).

\subsection{Fuzzy rough sets and similarity relations}

Rough sets can be extended by replacing an equivalence relation by a fuzzy similarity relation (Dubois and Prade 1990), thus introducing degrees of possibility and necessity that an element belongs to a given crisp set, due to the fuzzy granulation of the referential. There is an extensive literature on fuzzy rough sets (Radzikowska and Kerre 2002) that seems to be unrelated to the Höhle-Klawonn view of extensional fuzzy sets recalled above, that also relies on similarity relations, and induces a form of granulation of the referential. The bridge between fuzzy rough sets and extensional fuzzy sets is however made in Recassens (2010).

The notion of extensional fuzzy set with respect to a similarity relation clearly generalizes the notion of exact set in rough set theory, that is formed by the union of equivalence classes. The so-called extensional hull of a fuzzy set, viewed as the smallest extensional fuzzy set containing it, is formally the same as the upper approximation of this fuzzy set by means of the partition formed by the fuzzy singletons. In particular the extensional hull $\hat{X}$ of a set $X$ [of the form (11)] does coincide with the upper fuzzy approximation of set $A$ in the sense of fuzzy rough sets (Dubois and Prade 1990). In the theory of extensional fuzzy sets, the lower approximation of a fuzzy set $F$ takes the following form Boixader et al. (2000), Recassens (2010):

$$
F_{E}(u)=\inf _{u \in U} E(u, v) \rightarrow F(v)
$$

with a residuated implication $\rightarrow$ with respect to a t-norm $*$. $F_{E}$ is the largest extensional fuzzy set included in $F$, namely it is such that $F_{E}(u) * E(u, v) \leq F(v), \forall u \in U$. In other words, $F_{E}$ is of the form $E^{N}(F)$ in the sense of necessity functions. However, we do not have that $F_{E}=$ $\overline{\bar{F} \circ E}$ in general, which suggests that such approximation pairs may fail to have all properties of usual rough sets. This approach thus differs from Dubois and Prade (1990) where the chosen implication in (20) is Kleene's, so that the lower approximation is precisely defined by $\overline{\bar{F} \circ E}$, respecting the duality between upper and lower approximations, but possibly failing the extensionality property. The connection between extensionality and rough sets has been very recently discussed by Chakraborty (2011) in the setting originally described by Higgs (1973), that inspired Höhle and Klawonn, and in the fuzzy set setting in Recassens (2010), Chapter 3.

So, pairs $\left(F \circ E, F_{E}\right)$ can be viewed as fuzzy rough sets. They provide the approximate description of fuzzy sets by means of fuzzy points in the sense of a fuzzy similarity relation, just like rough sets in the more elementary setting of a crisp equivalence relation. In Ruspini (1991), and the literature on similarity-based reasoning (Godo and Rodriguez 2008), a fuzzy set is always understood as the extensional hull of a crisp set. The connections and difference of points of view between fuzzy rough sets and similarity-based reasoning after Ruspini, have already been emphasised (Dubois and Prade 1998b). While rough sets and granulation insist on the idea that elements of the referential cannot be distinguished, the idea of similarity, often then termed fuzzy equality, and viewed as the negative of a distance, insists on making a difference between elements however close they can be. If obeying separability, fuzzy similarity relations are then more tailored to interpolation purposes (Dubois et al. 1997; Perfilieva et al. 2012) than to classification.

\section{Concluding remarks}

The idea of granulation Zadeh (1997) is based on the notion of cluster whereby 
1. any pair of members of a cluster should be closely related in some sense;

2. any member of a cluster should be sufficiently separated from any member from outside the cluster.

The paper has provided a discussion of several areas, where the idea of granulation is central, and notions of closeness and separation can be defined. On this ground, similarities between different settings like possibility theory, formal concept analysis, extensional fuzzy sets, and rough sets have been laid bare. Similar structures were found to be at work in such settings. This kind of attempt may lead to mutual enrichments between theories, as in the parallel between possibility theory and formal concept analysis.

It is clear that such formal links should be further investigated in more general representation frameworks such as pattern structures (Ganter and Kuznetsov 2001; Assaghir et al. 2010), but also using algebraic structures beyond residuated lattices exploited in Bělohlávek (2002). Indeed, the many-valued FCA suffers from two limitations. First, one may object to the fact that most of the time, the negation in residuated lattice is not involutive, which may make the decomposition of fuzzy contexts into independent subcontexts more difficult: it may be difficult to write Eq. (17) in the form of Point 4 of Proposition 2. One way to do so is to interpret implication as $a \rightarrow b=n(a * n(b))$ in (17) for an involutive negation $n$. But then the underlying conjunction associated to $\rightarrow$ through residuation will no longer be associative nor commutative (Dubois and Prade 1984; Fodor 1991). A study of multivalued FCA using nonassociative, non-commutative conjunctions is carried out by Medina et al. (2009), using so-called multi-adjoint concept lattices. Lastly, it seems to be idealistic to assume that the degrees of satisfaction of all properties of objects can be measured on the same non-Boolean scale. This assumption may be problematic when processing real nonBoolean data. This issue is taken up at the theoretical level by Medina and Ojeda-Aciego (2012) using multi-adjoint concept lattices.

\section{References}

Assaghir Z, Kaytoue M, Prade H (2010) A possibility theory-oriented discussion of conceptual pattern structures. In: Deshpande A, Hunter A (eds) Scalable uncertainty management (SUM'10), Toulouse, ser. LNAI, vol 6379, pp 70-83. Springer, New York

Bellman RE, Kalaba R, Zadeh L (1966) Abstraction and pattern classification. J Math Anal Appl 13:1-7

Bellman RE, Zadeh L (1970) Decision making in a fuzzy environment. Manag Sci 17:B141-B164

Bělohlávek R (1999) Fuzzy Galois connections. Math Log Quart 45:497-504

Bělohlávek R (2002) Fuzzy relational systems: foundations and principles. Kluwer Academic/Plenum Publishers, New York
Bělohlávek R (2009) Optimal triangular decompositions of matrices with entries from residuated lattices. Int $\mathrm{J}$ Approx Reason 50:1250-1258

Boixader D, Jacas J, Recassens J (2000) Fuzzy equivalence relations: advanced material. In: Dubois D, Prade H (eds) Fundamentals of fuzzy sets: the handbooks of fuzzy sets series, Kluwer, Boston, Mass, 261-290

Chakraborty M (2011) On fuzzy sets and rough sets from the perspective of indiscernibility. In: Proceedings of 4th Indian conference on logic and its applications, Delhi, ser. LNAI, vol 6521, Springer, pp 22-37

Ciucci D, Dubois D, Prade H (2014) The structure of oppositions in rough set theory and formal concept analysis: toward a new bridge between the two settings. In: Foundations of intelligent knowledge systems (FoIKS 2014), Lecture Notes in Computer Science, Vol 8367, Springer, pp 154-173

Djouadi Y, Dubois D, Prade H (2010) Possibility theory and formal concept analysis: context decomposition and uncertainty handling. In: Hüllermeier E, Kruse R, Hoffmann F (eds) Computational intelligence for knowledge-based systems design. Proceeding of 13th international conference on information processing and management of uncertainty (IPMU 2010), Dortmund, ser. LNCS, vol 6178, Springer pp 260-269

Dubois D, Esteva F, Garcia P, Godo L, Prade H (1997) A logical approach to interpolation based on similarity relations. Int $\mathrm{J}$ Approx Reason 17(1):1-36

Dubois D, Prade H (1980) Fuzzy sets and systems: theory and applications. Academic Press, New York

Dubois D, Prade H (1984) A theorem on implication functions dened from triangular norms. Stochastica 8:267-279

Dubois D, Prade H (1988) Possibility theory: an approach to computerized processing of uncertainty. Plenum Press, Berlin

Dubois D, Prade H (1990) Rough fuzzy sets and fuzzy rough sets. Int J Gen Syst 17:191-209

Dubois D, Prade H (1992) Possibility theory as a basis for preference propagation in automated reasoning. In: Proceeding of 1st IEEE international conference on fuzzy systems 1992 (FUZZIEEE'92), San Diego, CA, pp 821-832, 8-12 March 2012

Dubois D, Prade H (1996) What are fuzzy rules and how to use them. Fuzzy Sets Syst 84:169-185

Dubois D, Prade H (1997) The three semantics of fuzzy sets. Fuzzy Sets Syst 90:141-150

Dubois D, Prade H (1998a) Possibility theory: qualitative and quantitative aspects. In: Gabbay D, Smets P (eds) Quantified representation of uncertainty and imprecision, ser. Handbook of defeasible reasoning and uncertainty management systems, Kluwer Acad. Publ, vol 1, pp 169-226

Dubois D, Prade H (1998b) Similarity versus preference in fuzzy setbased logics. In: Orlowska E (ed) Modelling incomplete information: rough set analysis, ser. Studies in fuzziness and soft computing. Heidelberg: Physica Verlag, pp 441-461

Dubois D, Prade H (2009) Possibility theory and formal concept analysis in information systems. In: Proceeding of international fuzzy systems association world congress and conference of the european society for fuzzy logic and technology (IFSAEUSFLAT'09), Lisbon, pp 1021-1026. 20-24 July 2009

Dubois D, Prade H (2012) Possibility theory and formal concept analysis: characterizing independent sub-contexts and handling approximations. Fuzzy Sets Syst 196:4-16

Dubois D, Prade H (2015) Possibility theory and its applications: where do we stand? In: Kacprzyk J, Pedrycz W (eds) Springer handbook of computational intelligence, Springer, pp 31-60

Dubois D, Dupin de Saint Cyr F, Prade H (2007) A possibiltytheoretic view of formal concept analysis. Fundam Inform 75(1-4):195-213 
Düntsch I, Orlowska E (1999) Mixing modal and sufficiency operators. Bull Sect L Polish Acad Sci 28(2):99-106

Düntsch I, Gediga G (2003) Approximation operators in qualitative data analysis. In: Theory and application of relational structures as knowledge instruments, pp 216-233

Fodor J (1991) On fuzzy implication operators. Fuzzy Sets Syst 42(3):293-300

Ganter B, Kuznetsov SO (2001) Pattern structures and their projections. In: ICCS '01: Proceedings of the 9th international conference on conceptual structures. Springer, pp 129-142

Ganter B, Wille R (1999) Formal concept analysis. Springer, New York

Gaume B, Navarro E, Prade H (2010) A parallel between extended formal concept analysis and bipartite graphs analysis. In: Hüllermeier E, Kruse R, Hoffmann F (eds) Computational intelligence for knowledge-based systems design, proceeding of 13th international conference on information processing and management of uncertainty (IPMU 2010), Dortmund, ser. LNCS, Springer, vol 6178, pp 270-280

Georgescu G, Popescu A (2004) Non-dual fuzzy connections. Arch Math L 43(8):1009-1039

Godo L, Rodriguez RO (2008) Logical approaches to fuzzy similarity-based reasoning: an overview. In: Preferences and similarities, ser. CISM courses and lectures. Springer 504:75-128

Höhle U (1988) Quotients with respect to similarity relations. Fuzzy Sets Syst 27:31-44

Higgs D (1973) A category approach to Boolean valued set theory. Tech. Rep., University of Waterloo, Canada

Jacas J (1990) On the generators of T-indistinguishability operators. Stochastica 12:49-63

Klawonn F (2000) Fuzzy points, fuzzy relations and fuzzy functions. In: Novák V, Perfilieva I (eds) Discovering the world with fuzzy logic. Physica-Verlag, Heidelberg, pp 431-453

Klement EP, Mesiar R, Pap E (2000) Triangular norms. Kluwer Academic, Dordrecht

Medina J, Ojeda-Aciego M (2012) On multi-adjoint concept lattices based on heterogeneous conjunctors. Fuzzy Sets Syst 208:95-110
Medina J, Ojeda-Aciego M, Ruiz-Calviño J (2009) Formal concept analysis via multi-adjoint concept lattices. Fuzzy Sets Syst 160(2):130-144

Pawlak Z (1981) Information systems theoretical foundations. Inf Syst 6(3):205-218

Pawlak Z (1991) Rough sets: theoretical aspects of reasoning about data. Kluwer Acad. Publ, Dordrecht

Perfilieva I, Dubois D, Prade H, Esteva F, Godo L, Hodáková P (2012) Interpolation of fuzzy data: analytical approach and overview. Fuzzy Sets Syst 192:134-158

Popescu A (2004) A general approach to fuzzy concepts. Math L Q $50: 265-280$

Radzikowska AM, Kerre E (2002) A comparative study of fuzzy rough sets. Fuzzy Sets Syst 126(2):137-155

Recassens J (2010) Indistinguishability operators, STUDFUZZ 260. Springer, Berlin

Ruspini EH (1991) On the semantics of fuzzy logic. Int J Approx Reason 5(1):45-88

Valverde L (1985) On the structure of F-indistinguishability operators. Fuzzy Sets Syst 17:313-328

Yao Y (2004) A comparative study of formal concept analysis and rough set theory in data analysis. In: Rough sets and current trends in computing, 4th international conference, RSCTC 2004, Uppsala, Sweden: LNCS 3066, Springer, pp 59-68. 1-5 June 2004

Yao YY, Chen Y (2006) Rough set approximations in formal concept analysis. Trans Rough Sets V LNCS 4100:285-305

Zadeh LA (1965) Fuzzy sets. Inform Control 8:338-353

Zadeh LA (1971) Similarity relations and fuzzy orderings. Inf Sci 3:177-200

Zadeh LA (1978) Fuzzy sets as a basis for a theory of possibility. Fuzzy Sets Syst 1:3-28

Zadeh LA (1997) Toward a theory of fuzzy information granulation and its centrality in human reasoning and fuzzy logic. Fuzzy Sets Syst 90:111-128

Zadeh LA (2005) Toward a generalized theory of uncertainty (GTU): an outline. Inf Sci 172:1-40 